Brit. J. vener. Dis. (1961), 37, 142.

\title{
DIFFICULTIES OF DIAGNOSIS AND TREATMENT OF GONORRHOEA IN YOUNG GIRLS*
}

\author{
BY \\ BOHDAN MICHALOWSKI \\ Warsaw
}

Since sulphonamides and especially antibiotics have been available, the treatment of gonorrhoea has presented no serious difficulty except in young girls before puberty in whom such therapy is often unsuccessful. The term "vulvo-vaginitis", often used to describe gonococcal infection in young girls, is misleading, as the gonococcus may invade not only the vagina but also the urethra, occasionally the rectum and, according to recent observations, the cervix. However, the vaginal mucosa is the most frequent site of infection. Gonorrhoea in young girls, which is very rare in Western Europe, unfortunately occurs more often in Poland, as shown in the Ministry of Health's statistics of the registered cases of gonorrhoea in young girls (Table).

TABLE

INCIDENCE OF GONORRHOEA IN GIRLS UP TO 14 YEARS, 1950-59

\begin{tabular}{c|c|c|c}
\hline \multirow{2}{*}{ Year } & \multicolumn{2}{|c|}{ Age (Yrs) } & \multirow{2}{*}{ Total } \\
\cline { 2 - 3 } & $0-3$ & $4-14$ & \\
\hline 1950 & 121 & 226 & 347 \\
1951 & 124 & 204 & 328 \\
1952 & 143 & 56 & 199 \\
1953 & 184 & 307 & 491 \\
1954 & 121 & 218 & 339 \\
1955 & 168 & 243 & 411 \\
1956 & 139 & 274 & 413 \\
1957 & 75 & 194 & 269 \\
1958 & 68 & 165 & 233 \\
1959 (1st quarter) & 49 & 61 & 110 \\
\hline
\end{tabular}

In Poland, cases of gonorrhoea in juveniles must be reported to the Health Service authorities. These data may exaggerate the true position through erroneous diagnosis, but the chronic character of this infection, its tendency to relapses, and its resistance to treatment, constitute a medical as well as a social problem in Poland.

* Paper read at the European Symposium of the I.U.V.D.T. at Cracow, Poland, in September, 1960.
Infected children must be isolated and as they are not allowed to attend nurseries, kindergartens, and schools they often miss a whole school year, and mothers have to give up work to take care of their infected children. The little girls usually acquire the infection within the family circle through sharing a bed with an infected mother or sister. Transmission of infection through toilet articles-towels, sponges, basins, etc.-is rarer, and contamination from baths occurs only occasionally. Infection in daynurseries and schools is also possible and here the detection of the contaminating agent is difficult. Infection by rape is extremely rare. Gonorrhoea is exceptional in rural communities but is much more frequent in towns especially among the poor classes with low standards of hygiene. Severe overcrowding due to the housing shortage, especially in towns, is another factor.

The vaginal mucosa of young girls is a favourable medium for gonococcal infection. In sucklings, the vaginal mucosa consists of a solid epithelium composed of numerous layers of cells containing keratohyaline and large quantities of glycogen; the vaginal reaction is acid and Döderlein bacilli may be found in the bacterial flora. As the child develops, further changes occur in the vaginal mucosa. The epithelium becomes more delicate, consisting of only a few layers of cells, glycogen disappears, and cocci replace the Döderlein bacilli. Thus the suckling child is the least susceptible to gonococcal infection, whereas little girls of 2 to 8 years of age may become infected very easily. This fact is supported by the numbers of registered cases shown in the Table. Although the lesions caused by the gonococcus are usually superficial, the infection in some cases penetrates deeper, through the epithelial cells, infiltrating the neighbourhood of sub-mucous glands or invading the glands themselves.

Three clinical forms (acute, subacute, and latent) may be distinguished. 
In the acute form, which is very rare, the onset is sudden. The labia majora becomes oedematous and covered with a purulent secretion, and the child complains of pain while urinating. Large numbers of intracellular gonococci are to be found in smears from the vaginal secretion.

In the subacute form, the pathological picture is more discrete and the signs and symptoms are less severe.

In the latent form, which is the most frequent, erythema and traces of dried secretion may be found between the labia especially if the child has not been bathed. The vaginal discharge is very discrete and may be purely mucous. The child usually feels well and it is only the traces of discharge on the child's underclothes which compel the parents to consult a physician. Lesions may also affect the external os or the cervix itself, and these are easily detected with the vaginoscope. The lips of the cervix may be swollen and there may be some secretion, or small raspberry-like granulations. These cervical lesions are prone to frequent relapse. The typical gonococcus is rarely found in smears and diagnosis is often impossible without cultures to distinguish the gonococci from other diplococci.

It is well known that the gonococcus grows with difficulty in artificial media. We use a medium, modified by Rzucidlo, composed of human or rabbit blood, casein hydrolysate, and a mixture of vitamins. The culture is incubated for 24 hours in an atmosphere containing 10 per cent. carbon dioxide, but even in this improved medium it is difficult to differentiate gonococci from microcolonies of saprophytes. While the gonococcus should ferment only glucose, in practice strains of gonococci may be grown after antibiotic treatment which give defective fermentation reactions. The oxydase test is useful only as an auxiliary method in differential diagnosis and it is desirable for the culture method to be used in special laboratories, with a highly qualified staff. We put the material taken from the patient directly into the Petri dish, since collecting and then sending the specimen to a laboratory does not give satisfactory results.

Neither the gonococcal complement-fixation test nor the cutaneous reaction to vaccine plays any role in the diagnosis of gonorrhoea.

The diagnostic difficulties encountered are equalled by those encountered in the treatment of juvenile gonorrhoea, especially in its latent form. At first, some cases were resistant only to sulphonamides, but now more and more cases are resistant to penicillin. Various methods of giving penicillin (e.g. injections of fractional daily doses several times a day to maintain the necessary penicillin blood level) and different forms of penicillin (e.g. crystalline, procaine penicillin, and depot preparations) have been tried, but penicillin alone is not sufficient to cure such cases. Streptomycin has remarkable activity against gonococci but in some cases of juvenile gonorrhoea it has failed. The results are better when penicillin is combined with streptomycin.

Oestrogen therapy was used before the discovery of sulphonamides and antibiotics and now we return to it if antibiotics fail. Side-reactions during oestrogen therapy are not alarming. Transient swelling of the breasts with development of a little pubic hair may occur, and the method should not be applied to older girls lest precocious menstruation results.

In spite of such treatment relapses often occur in cases of the latent form; they usually occur 2 to 3 months after the end of treatment but may occur even after 6 to 8 months. At the children's department of skın and venereal diseases of the Second Municipal Hospital in Warsaw, 224 cases of gonorrhoea in young girls have been observed. 139 cases developed between the ages of 3 and 7 years and 20 per cent. of them were of the acute or subacute form. Most of the children were treated with penicillin and streptomycin $(600,000$ units penicillin and $1 \mathrm{~g}$. streptomycin). The recovery rate was 81 per cent. but longer observation revealed 19 per cent. of relapses. The results with streptomycin alone were less satisfactory (73 per cent. of recoveries and 20 per cent. of relapses), but very good results were obtained with folliculin (89 per cent. of recoveries).

In the latent form, local treatment was often required. Relapse and re-infection cannot be differentiated by clinical and laboratory examinations, and follow-up tests of cure must be rigorous. When treatment is finished, a few negative smears at intervals of a few days as well as three negative cultures are obligatory before the patient is discharged. The last clinical examination also includes vaginoscopy.

After leaving the hospital, the children are examined every fortnight for the first 3 months, once a month during the next 3 months, and then less frequently during the following 6 months. In addition, they are not admitted to day nurseries, kindergartens, or schools for 6 weeks after discharge from the hospital.

It is evident that gonorrhoea in young girls presents many difficulties. Diagnosis depends upon laboratory tests, the fermentation of glucose being the only specific one. The difficulty is increased by the fact that the pattern of fermentation may change under the influence of antibiotics. The other bacteriological and serological tests are not specific. To 
improve this situation, clinicians need the help of bacteriologists to develop better and easier tests.

\section{Summary}

The considerable incidence of gonorrhoea in young girls presents a difficult medical and social problem in Poland. The latent form is the most frequent and its diagnosis and treatment are often difficult. Good results were obtained by the application of hormones with penicillin. However, in spite of such methods of treatment, some relapses occur. Improved cultures and specific gonococcal tests, which could be carried out in ordinary laboratories, are needed.
Difficultés dans le diagnostic et le traitement de la blennorragie chez les jeunes filles jusqu'à 14 ans

\section{Résumé}

Le nombre élevé des cas de la gonorrhée infantile en Pologne constitue d'après l'auteur un problème aussi bien médical que social. La difficulté du traitement de la forme latente qui est aussi la forme la plus fréquente est causée par la résistance au traitement par la penicilline.

De bons résultats ont été obtenus en appliquant la pénicilline et la folliculine. Malgré ces différents traitements on observe des rechutes. Il est nécessaire d'améliorer les méthodes de culture afin de trouver des moyens de diagnostic qui seront plus faciles à exécuter dans les laboratoires ordinaires. 